\title{
Oxidative Phosphorylation Inhibitor IM156
}

National Cancer Institute

\section{Source}

National Cancer Institute. Oxidative Phosphorylation Inhibitor IM156. NCI Thesaurus.

Code 153372.

An orally bioavailable biguanide compound and mitochondrial oxidative phosphorylation

(OxPhos) inhibitor, with potential antineoplastic activity. Upon administration, IM156

inhibits oxidative phosphorylation, decreases mitochondrial function, prevents tumor cell

metabolism and deprives tumor cells of energy, thereby preventing tumor cell

proliferation. Mitochondrial OxPhos is overactivated in cancer cells and plays a key role in tumor cell proliferation. Drug resistant tumor cells are very susceptible to decreased mitochondrial OxPhos as they cannot easily compensate for the decrease in mitochondrial function by increasing glycolysis. 